\title{
Research on impact factor of the HPA power synthesizes
}

\author{
Cheng Gang, Dai Chao, Liu Shuai
}

\section{China Satellite Maritime Tracking and Control Department, Jiangyin, 214431, China}

\begin{abstract}
In aerospace measurement and control systems, solid-state power amplifier as an important part of the monitoring and control stations for the monitoring station to launch the carrier to provide high enough power to meet the required system EIRP. In this paper, the basic concept and principle of the transmission line are analyzed, and the method of solving the problem is put forward according to the actual situation of the solid - state power amplifier of the transmitting sub - system: 1 ). Based on the transmission line impedance matching technology, the transmission line impedance mismatch problem is solved. 2). Add phase shift components, to solve the transmission line between the phase match inconsistencies.
\end{abstract}

Key words: Reflection power; transmission line; impedance matching

\section{Introduction}

A model of continuous-wave S-band solid-state power amplifier for high-power experiments, A set of solid-state amplifier of a single amplifier box there is too much power to reflect the problem. A set of solid-state power amplifier is the power amplifier box A1, power amplifier box A2, power amplifier box A3 and amplifier box A4 four separate amplifier box, the maximum output power of each amplifier box $300 \mathrm{~W}$, four power amplifier box The output power is synthesized by the synthesizer, and the maximum output power is $800 \mathrm{~W}$. From the analysis of the empirical data, it can be seen that the total reflected power is relatively small after the synthesis of the synthesizer, and the reflection power of the single power amplifier box is obviously larger.

\section{Basic principles and key technologies}

1.1 Basic Equation of Transmission Line and Its Solution

Uniform lossless transmission line equation:

$$
\begin{aligned}
& U(z)=A_{1} e^{-j \beta z}+A_{2} e^{j \beta z} \\
& I(z)=\frac{1}{Z_{0}}\left(A_{1} e^{-j \beta z}-A_{2} e^{j \beta z}\right)
\end{aligned}
$$

Usually the load side of the transmission line is given, so there are boundary conditions: the known terminal voltage and current, then get the transmission line at any point of the instantaneous voltage and transient current is:

$$
\begin{aligned}
& u(z, t)=\left|A_{1}\right| \cos \left(\omega t+\psi_{1}-\beta z\right)+\left|A_{2}\right| \cos \left(\omega t+\psi_{2}+\beta z\right) \\
& i(z, t)=\frac{\left|A_{1}\right|}{Z_{0}} \cos \left(\omega t+\psi_{1}-\beta z\right)-\frac{\left|A_{2}\right|}{Z_{0}} \cos \left(\omega t+\psi_{2}+\beta z\right)
\end{aligned}
$$

In summary, we can draw the conclusion: uniform lossless transmission line voltage and current, under normal circumstances is more than two phase velocity in the opposite direction of the spread of the sine wave superposition; from the power supply to the load wave propagation is called the incident wave, The wave from the load to the power source is called the reflected wave; the ratio of the voltage and the current of the two waves is a constant value, which is the characteristic impedance of the transmission line.

\subsection{Analysis of Transmission Line Working State}

In order to describe the reflection of the wave, the reflection coefficient is introduced. The equations (3) and (4) can be written as:

$$
U(d)=U^{+}(d)+U^{-}(d)=U^{+}(d)[1+\Gamma(d)]
$$




$$
\begin{aligned}
& I(d)=I^{+}(d)+I^{-}(d)=I^{+}(d)[1-\Gamma(d)] \\
& Z(d)=Z_{0} \frac{1+\Gamma(d)}{1-\Gamma(d)}
\end{aligned}
$$

In the terminal:

$$
Z_{l}=Z_{0} \frac{1+\Gamma_{l}}{1-\Gamma_{l}}
$$

This equation shows that there is a one-to-one relationship between the input impedance at any point on the transmission line and the reflection coefficient at that point. According to the nature of the load impedance, the transmission line has the following three different working conditions, followed by the analysis of these three working conditions.

\subsection{Traveling wave state (no reflection)}

From formula (3) and formula (4), we can see that the condition of no reflection wave is Z1 = Z0, that is, load matching. In this case,

$$
\begin{aligned}
& U(\mathrm{z})=\frac{U_{0}+Z_{0} I_{0}}{2} e^{-j \beta z}=\mathrm{U}_{0}^{+} e^{-j \beta \mathrm{z}} \\
& \mathrm{I}(\mathrm{z})=\frac{U_{0}+Z_{0} I_{0}}{2 Z_{0}} e^{-j \beta z}=\mathrm{I}_{0}^{+} e^{-j \beta \mathrm{z}} \\
& \mathrm{Z}(\mathrm{z})=\mathrm{Z}_{0}
\end{aligned}
$$

It can be seen that the characteristics of the traveling wave state are: 1) .Voltage, current amplitude of each point along the line without consumption; 2). The voltage and current phases at each point along the line continuously increase with z, the phase of voltage and current are the same; The input impedance of each point is equal to the characteristic impedance of the transmission line.

\subsection{Standing wave state (total reflection)}

a. Terminal short

Along the line voltage and current can be obtained:

$$
\begin{aligned}
& \mathrm{U}(\mathrm{d})=j 2 \mathrm{U}_{1}^{+} \sin \beta d \\
& \mathrm{I}(\mathrm{d})=\frac{2 \mathrm{U}_{1}^{+}}{\mathrm{Z}_{0}} \cos \beta d \\
& \mathrm{Z}_{\mathrm{in}}^{\mathrm{sc}}(\mathrm{d})=\mathrm{j} \mathrm{Z}_{0} \operatorname{tg} \beta d
\end{aligned}
$$

It can be seen that the magnitude of the voltage and current along the short-circuit of the terminal is different with the position. The input impedance of each point along the line is pure reactance and varies with frequency and distance. When the frequency is constant, the impedance varies with the distance period.

b. Terminal open line

Along the line voltage, current and input impedance can be obtained as:

$$
\mathrm{U}(\mathrm{d})=j 2 \mathrm{U}_{1}^{+} \cos \beta d
$$




$$
\begin{aligned}
& \mathrm{I}(\mathrm{d})=\frac{2 \mathrm{U}_{1}^{+}}{Z_{0}} \sin \beta d \\
& \mathrm{Z}_{\mathrm{in}}^{\mathrm{oc}}(\mathrm{d})=-\mathrm{j} \mathrm{Z}_{0} \operatorname{ctg} \beta d
\end{aligned}
$$

From the above analysis we can see that the characteristics of the standing wave: 1 ). The voltage and current at each point along the time and distance position have $90^{\circ}$ phase difference, so the standing wave state, the line neither energy loss, energy. 2). The voltage (or current) at each point along the line on both sides of the node is inverted, and the voltage (or current) is in phase with each other between adjacent nodes.

1.5 Line standing wave state (partial reflection case)

When the transmission line termination connected to the general impedance, the terminal reflection coefficient:

$$
\Gamma_{1}=\frac{Z_{1}-Z_{0}}{Z_{1}+Z_{0}}=\left|\Gamma_{1}\right| e^{ \pm j \phi 1}
$$

At this time the line voltage:

$$
U(d)=\mathrm{U}_{1}^{+}\left(1-\Gamma_{1}\right) e^{j \beta d}+2 \Gamma_{1} \mathrm{U}_{1}^{+} \cos \beta d
$$

This indicates that the line voltage that there is a traveling wave component and standing wave component, it is called standing wave.

\section{Way of solving the problem}

\subsection{Impedance matching of transmission lines}

Transmission line in the traveling wave state of the loss is small, high efficiency, the standing wave at this time the smallest power to bear the maximum capacity. However, transmission lines generally do not operate in the traveling wave state. This requires the addition of matching devices, so that the transmission line in the traveling wave state, this is the so-called impedance matching. The method is to add a matching device between the load and the transmission line. The basic requirement of the matching device is to introduce the additional loss as small as possible, the frequency band as wide as possible and can adapt to various load. Index line matching method can meet the requirements.

To match the transmission line to an exponential line, the transmission line characteristic impedance needs to be satisfied:

$$
\mathrm{Z}_{\text {in }}=\frac{\mathrm{Z}_{0}(0)}{-\mathrm{j} \frac{\delta}{2 \beta}+\sqrt{1-\left(\frac{\delta}{2 \beta}\right)^{2}}} \mathrm{e}^{\delta \mathrm{l}}=\mathrm{Z}_{\mathrm{l}} \mathrm{e}^{\delta \mathrm{l}}
$$

In this case, the load impedance is equal to the characteristic impedance of the exponential terminal, and the characteristic impedance at the beginning of the exponential line is equal to the characteristic impedance of the main transmission line and matched at the same time.

\subsection{Transmission line phase matching}

Power synthesis efficiency depends not only on the performance of the synthesizer itself, but also on the amplitude and phase of the synthesized signal. For an n-way fully symmetrical synthesizer, its synthesis efficiency in each input signal amplitude and phase of the situation is fully consistent with the maximum. In reality, the synthesizer is not ideal, the amplitude and phase of each signal cannot be exactly the same. According to engineering practice experience, when the phase difference is less than $20^{\circ}$, the effect on the synthesis efficiency is less than $2 \%$. 
In order to ensure the consistency of two-phase signal phase shift must be used technology. There are many ways to phase-shift, change the length of the cable can be phase-shifted, insert phase shifter can also change the phase relationship.

Using the vector network analyzer, measured the solid-state power amplifier A sets of various input cables, power amplifier box, the output cable phase is as follows:

Table 1. Initial Phase Data Table

\begin{tabular}{|c|c|c|c|c|}
\hline Number & Input cable & Amplifier box & Output cable & Total phase value \\
\hline 1 & $124^{\circ}$ & $54^{\circ}$ & $-85^{\circ}$ & $93^{\circ}$ \\
\hline 2 & $145^{\circ}$ & $56^{\circ}$ & $-84^{\circ}$ & $117^{\circ}$ \\
\hline 3 & $130^{\circ}$ & $68^{\circ}$ & $-74^{\circ}$ & $124^{\circ}$ \\
\hline 4 & $136^{\circ}$ & $77^{\circ}$ & $-86^{\circ}$ & $125^{\circ}$ \\
\hline
\end{tabular}

Can be seen, four amplifier box between the phase difference is large, resulting in a reflection of the phenomenon of excessive power; the specific reflected power can be seen in Table 1. In view of the above, we will input cable, amplifier box, output cable for re-combination, the combination of the results in the following table:

Table 2. Adjusted phase data table

\begin{tabular}{|c|c|c|c|c|}
\hline Number & Input cable & Amplifier box & Output cable & Total phase value \\
\hline 1 & A1 $\left(124^{\circ}\right)$ & A4 $\left(77^{\circ}\right)$ & A4 (-86 $)$ & $115^{\circ}$ \\
\hline 2 & A2 $\left(145^{\circ}\right)$ & A2 $\left(56^{\circ}\right)$ & A1 $\left(-85^{\circ}\right)$ & $116^{\circ}$ \\
\hline 3 & A3 $\left(130^{\circ}\right)$ & A3 $\left(68^{\circ}\right)$ & A2 (-84 $)$ & $114^{\circ}$ \\
\hline 4 & A4 $\left(136^{\circ}\right)$ & A1 $\left(54^{\circ}\right)$ & A3 (-74 $)$ & $116^{\circ}$ \\
\hline
\end{tabular}

At this time, the reflected power of each box decreased significantly, the maximum reflected power of only 5\%. It can be seen that phase matching technology can effectively improve the reflection power is too large, the phase of the case, the reflected power is small, the larger the phase difference, the reflected power larger. In addition to re-combination of input cables, power amplifier box, the output cable can be achieved phase matching, It is also possible to install a phase shifter on the transmission line of each amplifier box, and adjust the phase shifter so that the transmission line phase returns to a consistent state whenever the phase on the transmission line changes.

\section{Summary}

In this paper, through a type of continuous-wave S-band solid-state power amplifier mechanism analysis, summed up the reflected power is too large due to: impedance matching and phase inconsistencies. It is found that exponential line method can solve the problem of impedance matching very well. Phase shifter method can solve the problem of phase inconsistency well. Transmission line phase inconsistency of the effective solution to a certain extent, reflected power has been greatly improved. Phase matching method has been used in practice, and achieved good results for the future due to phase matching inconsistency caused by solid-state power amplifier reflected power is too large a similar problem, provides a good reference.

\section{Reference:}

[1] DeLisio M P, Robert A Y. Quasi-optical and spatial power combining. IEEE Transactions on Microwave Theory and Techniques, 2012; 50 (3): 929-935

[2] Yan Zhigang. RF power synthesis technology. Inner Mongolia Radio and Television Technology, 2013; 4 (2):

32-37

[3] RFPA. RF20252120-800 S-Band Solid State Power Amplifier, 2006: 47 